\title{
ZEEMAN OBSERVATIONS OF THE MAGNETIC FIELD IN THE GALACTIC CENTRE.
}

\author{
U.J. Schwarz \\ Kapteyn Laboratory, P.0. Box 800 \\ 9700 AV Groningen \\ The Netherlands
}

\author{
J. Lasenby \\ Cavendish Laboratory \\ Cambridge CB3 OHE \\ England
}

ABSTRACT. Direct measurements of the magnetic field in the neutral ring at the Galactic centre were made by observing the Zeeman splitting in $H I$-absorption around $\pm 100 \mathrm{kms}^{-1}$ and give a field of $(-0.5 m G \pm 0.17 m G)$; significantly less than inferred from IR polarisation observations (Werner et al., 1988).

\section{Introduction.}

The Galactic centre shows a complex structure where one suspects that magnetic fields play an important role; especially in the parallel filaments of the "arc" and in the inclined ring of gas and dust between radii $\sim 2$ and 10pc (also known as the circum-nuclear disk or CND) surrounding $\mathrm{Sgr} \mathrm{A}^{*}$. Here we discuss this ring which is in almost circular motion with a velocity of $\sim 100 \mathrm{kms}^{-1}$. Evidence for this feature comes from observations of molecules, neutral hydrogen and ionized gas (for a review see e.g. Genzel and Townes, 1987). The ring is also seen in the far IR (Becklin et al. 1982). Werner et al. 1988 found polarisation at $100 \mu$ (up to $2 \%$ ) indicating a regular field along the ring. They put forward that if the magnetic pressure equals the turbulent gas pressure $\left(\Delta v=50 \mathrm{kms}^{-1}\right)$ a field strength of $10 \mathrm{mG}$ results.

\section{Zeeman $\mathrm{H}$-absorption WSRT synthesis observations.}

The observations were carried out in 1987 in two lots of $5^{h}$, resulting in a beam of $12 \mathrm{x}$ $50^{\prime \prime}$. A total range of $500 \mathrm{kms}^{-1}$ was observed with a velocity resolution of $7 \mathrm{kms}^{-1}$ after Hanning smoothing; the pixel increment is $4.1 \mathrm{kms}^{-1}$. The correlation of perpendicular feeds give the circular polarized flux (Stokes quantity V). The absorbed line flux $I$ can be derived by subtracting the continuum from the line maps. The $I$ maps were CLEANed. The magnetic field can be found by $B_{/ /}=3.4 V /(d I / d v)$ with $B_{/ /}$the component of the magnetic field along the line of sight in $m G, v$ and $I$ in $m J y, V$ the velocity in $k m s^{-1}$, see for more details e.g. Schwarz et al. 1986.

\section{Results.}

Among the various absorption components, there are two at $\pm 100 \mathrm{kms}^{-1}$ which can be associated with the ring structure (see e.g. Lewtas, 1987). The mean position of these (integrated over $20 \mathrm{kms}^{-1}$ ) are shown together with the IR data in Fig. 1 as asterisks falling on the 'edge' of the ring.

The $d I / d v=I^{\prime}$ spectrum can be derived by computing $(I(v+\Delta v)-I(v-\Delta v)) / 2 \Delta v$. The average $I^{\prime}$ - and $V$-spectra of the two features are shown in Fig. 2. The rms noise of $I^{\prime}$ is $0.1 \mathrm{mJy} \mathrm{kms} / \mathrm{s}^{-1}$ of $V=1.0 \mathrm{mJy}$, shown on both sides in Fig. 2. The -100 $k m s^{-1}$ feature is blended by a peak at $-80 \mathrm{kms}^{-1}$, therefore only the left part of the $I^{\prime}$ spectrum is useable. No significant peak in $V$ is found; from this an upper limit of $1.5 m G$ can be 
derived ( 3 times the rms). On the other hand at $+100 \mathrm{kms}^{-1}$ both the positive and negative gradients of the $I$ are strong and there is a corresponding run of $V$. From this we tentatively derive a field of $B_{/ /}=(-0.5 \pm 0.17) m G$.

\section{Discussion.}

The derived strength and/or upper limit of the magnetic field observed here is in conflict with the results from the IR polarisation $(10 \mathrm{mG})$. Since the field as derived by the polarization data is along the ring, the maximum line of sight component of the field should be found at the 'edges' of the ring. Either the strength of the magnetic field is overestimated when one equates magnetic and kinetic pressure or the spatial relationship between HI-absorption and IR ring is not valid.

Acknowledgements. We thank the staff of NFRA which runs the WSRT for the observations and excellent calibration. We also thank J. van Gorkom for her interest in this project.

\section{References:}

Becklin, E.E., Gatley, I., Werner, M.W. 1982, Ap. J. 258, 135

Genzel, R., Townes, C.H., 1987, Ann. Rev. Astr. Ap., 25, 277

Lewtas, J., 1986, Thesis, Cambridge

Schwarz, U.J., Troland, T.H., Albinson, J.S., Bregman, J.D., Goss, W.M.,

Heiles, C., 1986, Ap. J. 301, 320

Werner, M.W., Davidson, J.A., Morris, M., Novak, G., Platt, S.R.,

Hildebrand, R.H., 1988, Ap. J. 333, 729

Fig.1 $100 \mu$ polarisation results of Werner et al, 1988 with the

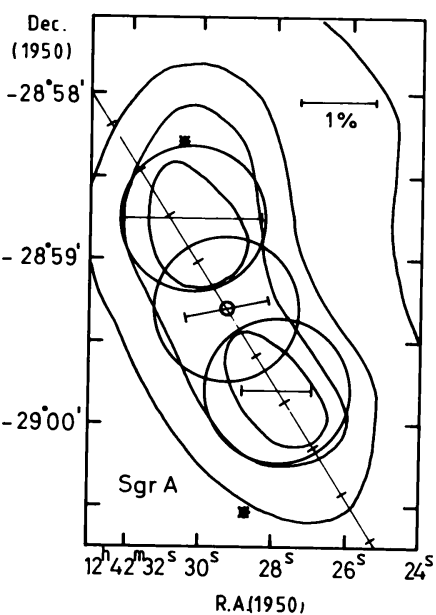
continuum data by Becklin et al, 1982). 0 is $\mathrm{SgrA}^{*}$ and the asterisks give the average position of the H-absorption features at $+100 \mathrm{kms}^{-1}$ (top) and $-100 \mathrm{kms}^{-1}$ (bottom).

Fig. $2 V$ and $d I / d v$ spectra with intensity scales indicated at the left and the right respectively. The rms errors are given as a bar for both.
a. $-100 \mathrm{kms}^{-1}$ feature,

b. $+100 \mathrm{kms}^{-1}$ feature.

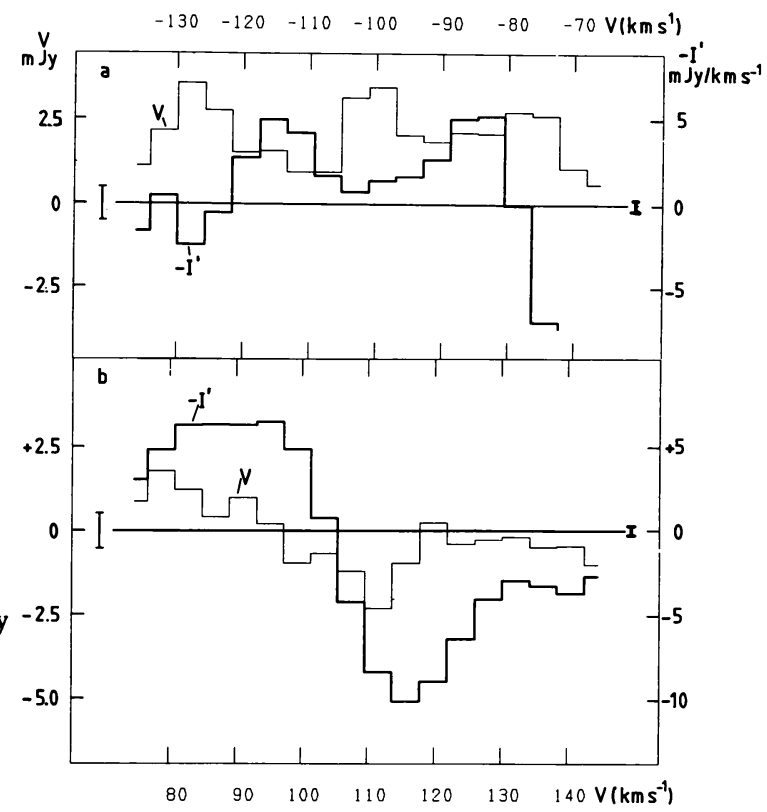

